\title{
A Systematic Review Protocol to Establish Plasmodium Falciparum Genetic Diversity, Multiplicity of Infection, Genotyping Approaches, and Methods of Reporting It in Africa.
}

\author{
Alex Mwesigwa ( $\nabla$ mwesigwaalex@gmail.com ) \\ Kabale University School of Medicine https://orcid.org/0000-0002-7544-3872 \\ Joaniter Immaculate Nankabirwa \\ Makerere University School of Medicine \\ Samuel Lubwama Nsobya \\ Makerere University College of Health Sciences \\ Charles Karamagi \\ Makerere University School of Medicine \\ Barbara Castelnuovo \\ IDI: Makerere University Infectious Diseases Institute \\ Pauline Byakika-Kibwika \\ Makerere University School of Medicine
}

\section{Protocol}

Keywords: Plasmodium falciparum, Genetic diversity, Multiplicity of infection, Genotyping and Africa

Posted Date: August 10th, 2021

DOl: https://doi.org/10.21203/rs.3.rs-764361/v1

License: (c) This work is licensed under a Creative Commons Attribution 4.0 International License.

Read Full License 


\section{Abstract}

\section{Background}

$P$. falciparum is the most important Plasmodium species that causes high malaria morbidity and mortality. The distribution of diverse and multiple $P$. falciparum infections is a major setback to the control and eventual elimination of malaria globally. Little efforts have been made to systematically establish P. falciparum genetic diversity and multiplicity of infection (MOI) in African settings.

Additionally, the choice of an effective $P$. falciparum genotyping approach for a specific endemic setting remains a challenge. The review aims to establish $P$. falciparum genetic diversity $\mathrm{MOl}$, genotyping approaches, and methods of reporting it in Africa. This will aid the evaluation of the impact of malaria control interventions and development of new control strategies.

Methods

The review will consider Randomized Clinical Trials (RCTs), Quasi-experiments, Cross-sectional studies, Cohort and Case-control studies about $P$. falciparum genetic diversity and $\mathrm{MOI}$. A literature search will be conducted in PubMed, Google scholar and MEDLINE databases for articles published from January 2010 to December 2020. Articles will be screened using Preferred Reporting Items for Systematic Reviews and Meta-Analysis (PRISMA) guidelines. Risk of bias in RCTs will be assessed using Cochrane risk of bias assessment tool while New Castle Ottawa tools will be used to assess the risk of bias in observational studies. Publication bias will be assessed using a funnel plot as well as Begg and Mazumdar's rank correlation test or Egger's test. The Higgins $\left.\right|^{2}$ statistic and Galbraith plots will be used to assess heterogeneity. A meta-regression analysis will be performed to explain the low heterogeneity.

\section{Discussion}

The findings from the study will enhance our understanding of the distribution and dynamics of $P$. falciparum genetic diversity and $\mathrm{MOI}$. This will provide insights to the changing landscape of malaria transmission and evaluation of malaria control interventions. Findings will also serve as baseline data for future studies on parasite population structure and antimalarial drug resistance surveillance across African countries.

\section{Background}

Malaria is a febrile illness that remains one of the major causes of morbidity and mortality globally. It is estimated that malaria accounted for 229 million cases and 409,000 deaths in 2019 (WHO, 2020). The WHO African Region carries a disproportionately high share of the global malaria burden with the majority $(99.7 \%)$ of the malaria infections being caused by $P$. falciparum (WHO, 2018, 2020).

Individuals living in areas of high malaria transmission intensity can concurrently be infected with multiple and genetically distinct clones of $P$. falciparum resulting in MOI (Kidima \& Nkwengulila, 2015; 
Sondén et al., 2015). Genetically diverse and multiple $P$. falciparum infections occur in both asymptomatic (Abamecha et al., 2020) and symptomatic (Soulama et al., 2009) malaria patients. P. falciparum genetic diversity and $\mathrm{MOI}$ enhance parasite virulence, malaria pathology and evasion of the immune response (Jensen, Adams, \& Hviid, 2020; Josling \& Llinás, 2015; Sondo et al., 2019). However, little effort has been made to systematically assess $P$. falciparum genetic diversity and $\mathrm{MOI}$ across a wide range of studies. Additionally, the choice of an effective $P$. falciparum genotyping approach for a specific endemic setting remains a challenge.

Characterization of $P$. falciparum genetic diversity and $\mathrm{MOI}$ helps to determine if leftover residual historical parasite lineages contribute to local malaria transmission or new parasite lineages migrating from other regions contribute to current malaria transmission (Carter et al., 2015; Landier et al., 2018; Niang et al., 2017). It also helps to determine gene flow dynamics and provides insight into the appropriate malaria control interventions (Nabet et al., 2016; Taylor et al., 2017; Wesolowski et al., 2018). Thus, this review aims to assess the $P$. falciparum genetic diversity, $\mathrm{MOI}$, genotyping approaches and methods of reporting it in Africa.

\section{Methods}

Review question. The systematic review aims to 1 ) Determine $P$. falciparum genetic diversity and $\mathrm{MOI}$ among malaria-infected individuals in Africa. 2) Identify the genotyping approaches and methods of reporting $P$. falciparum genetic diversity and $\mathrm{MOI}$ in Africa.

Table 1. The PICOST framework.

\begin{tabular}{|ll|}
\hline Population & Children and adults \\
\hline Intervention/Exposure & P. falciparum infection \\
\hline Comparator & None \\
\hline Setting & $\begin{array}{l}\text { Primary outcome: } P \text {. falciparum genetic diversity and multiplicity of } \\
\text { infection. } \\
\text { Secondary outcome: Genotyping approaches and methods of reporting } P . \\
\text { falciparum genetic diversity and MOI. }\end{array}$ \\
\hline Time & Africa \\
\hline
\end{tabular}

\section{Definition of the study outcome.}

$P$. falciparum genetic diversity is the variation in the genetic composition within $P$. falciparum species as a result of high genetic recombination rates (Jiang et al., 2011). 
Multiplicity of infection (MOI) is the number of distinct parasite clones simultaneously infecting an individual (Henning et al., 2004; Kiwanuka, 2009).

The systematic review will be conducted following the PRISMA guides as per the flow diagram below.

\section{Protocol registration.}

The protocol has been submitted for registration with PROSPERO (https://www.crd.york.ac.uk/prospero/). Acknowledgment receipt number 267661.

\section{Determining eligibility criteria.}

\section{Inclusion criteria.}

The study will include studies that report on $P$. falciparum genetic diversity and $\mathrm{MOI}$, detailing the genotyping approaches and reporting methods among individuals of all age groups, gender and malaria severity in African malaria transmission settings. Studies reporting on other Plasmodium species in addition to $P$. falciparum will only be included if the findings are stratified based on species. Both Randomized clinical trials (RCTs), Quasi-experiments, Cross-sectional studies, Cohort and Case-control studies will be included in this review. Also, multi-country studies will be considered if the results are stratified and it is clear which findings are from the African countries. We will only include articles published in English from January 2010 to December 2020 to capture the latest data.

\section{Exclusion criteria.}

Articles generated from studies conducted in areas whose malaria transmission level cannot be established, articles in which studies were conducted in or using animal models. Also, case reports, case series, conference deliberations, policies, opinions and editorials will not be included in this review. Articles whose full text cannot be retrieved will also be excluded.

\section{Information sources.}

The review will use three databases namely: PubMed, MEDLINE and Google scholar.

\section{Search strategy.}

The literature search will be conducted by two reviewers using Medical Subject Heading (MeSH) terms generated from PubMed. A pilot search in PubMed has yielded the following MeSH terms: 'Plasmodium falciparum', 'P. falciparum', 'Plasmodium falciparum malaria', 'Plasmodium parasites', 'Genetic diversity', 'Genetic Variations', 'Genetic differences', 'Different genotypes', 'Genotyping', 'Genotyping Technique*', 'Genotype Assignment Methodology', 'Genotype Calling Methods', 'Genotype Determination Methods', 'Methods, Genotype Determination', 'Africa'. The related MesH terms will be conjoined with the Boolean operator "OR" and the unrelated MesH terms will be conjoined with the Boolean operator "AND" to form a search string. The search string will be applied in all databases namely PubMed, Google scholar and 
MEDLINE to search for the articles. In each search, the MeSH terms 'Plasmodium falciparum', 'Genetic diversity' and 'multiplicity of infection' will be used to identify articles where they are included in either the title and or abstract. The search will be restricted to articles published in the English language from January 2010 to December 2020. Additional literature will be found through targeted search from references of included studies and grey literature as summarized below in the flow chart.

The articles/citations obtained from the three databases will be exported to EndNote X9. In EndNote, duplicates will be removed before the screening.

\section{Screening of articles.}

The searched articles will be assessed for the outcomes of interest in any African country. The screening process will be guided by the updated PRISMA screening guidelines (Page et al., 2021). The Articles will undergo Title and Abstract screening and those that pass these criteria will be considered for Full-text screening. The articles that have undergone Full-text screening will be the ones to include in this review. The screening will be done in duplicates by two independent reviewers. Disagreements will be resolved through discussion between a pair of other independent reviewers to reach a consensus.

\section{Data extraction.}

Data extraction will be done on articles that have passed full-text screening criteria by three reviewers using a form adapted from Cochrane collaboration (Appendix I). The data extraction form will capture information on; author, year of publication, country of study, study design, study setting, study population and sample size, and study review outcomes namely 1 ) $P$. falciparum genetic diversity, $\mathrm{MOI}$ and 2) Genotyping approaches used. The extracted data will be independently cross-checked by two reviewers.

\section{Risk of bias assessment.}

Three reviewers will independently assess the articles to minimize bias.

Cochrane risk of bias assessment tools (Higgins et al., 2011) will be used for assessment and judgment of risk of bias in RCT (Appendix II). The New Castle Ottawa tools will be used for Case-control, Observational Cohort, and Cross-Sectional Studies to critically appraise the selected studies. Both individual studies' bias, overall bias as well as publication bias will be assessed.

For assessment of bias in individual studies, we will establish the study design and outcome of each study, then compare it with appropriate preselected criteria for that study design and each predetermined outcome. This will enable us to make a judgment about the risk of bias for study design and the outcome in each study. Assessment of overall bias will be agreed upon by three independent reviewers using a modified ACROBAT-NRSi (A Cochrane Risk Of Bias Assessment Tool) Appendix III, clearly documenting and justifying all processes and decisions taken. Any differences will be resolved and then record the final rating for each outcome documenting the reasons for the judgment and process for the final judgment. The risk of bias will be categorized as Low, Moderate, Critical, or Serious risk of bias. 
Publication bias will be assessed using a funnel plot. Begg and Mazumdar's rank correlation test or Egger's test will be determined to statistically assess publication bias. If publication bias is detected, it will be corrected using the trim-and-fill method using Comprehensive Meta-Analysis software. We will also consider trials or protocol registries to check self-reporting bias

\section{Quality of evidence assessment.}

This will be ascertained by two independent reviewers using grading of recommendations, assessment and development and evaluations (GRADE) guidelines (Schünemann, Brożek, Guyatt, \& Oxman, 2013). Quality of evidence will be assessed based on five domains including; 1) Study design and limitations. This will be assessed by examining the risk of bias in individual studies by examining the risk of bias associated with study participation, study attrition, measurement of risk factor, outcome measurement, confounding measurement and analysis. 2) Inconsistency in selected articles. For this, the assessment will be based both on our judgment and confidence intervals of primary studies to determine variability/heterogeneity in results across studies. 3) Indirectness of the evidence. This will be assessed by verifying that the study population, risk factors and study outcomes fully represent the review question.4) Imprecision. This will be checked by taking note of the sample size calculation and rationale for it. The more the participants in the study the more precise the study is. Also, we will take note of heterogeneity and confidence interval around the estimated effect size across studies to ensure that it is not excessively wide. Our judgment of the overall precision will be based on the precision of results within each study taking into account the number of participants involved. We will aim to include in the review majority of precise studies. 5) Publication bias. This will be assessed by ensuring that any determinant factor of the study outcome has been investigated and reported by a large number of studies. Overall, the quality of evidence will be categorized as high, medium low or very low and documented using a modified grade quality of evidence assessment tool (Appendix IV).

\section{Data synthesis.}

Before data synthesis, we will first check to ensure the findings from the individual studies have been collated correctly. We will then summarize the characteristics of individual studies based on PICOST. These characteristics will be entered into a fresh table similar to the one of the data extraction form (Appendix I). Then we will compare these characteristics a cross studies to determine which studies are similar enough to be grouped or form a comparison group. After grouping similar studies, we will examine what data are available for synthesis. We will then tabulate study characteristics to present a summary of characteristics for comparison and analysis across studies using a fresh form (Appendix I). This will enable us to identify variations in the interventions which may lead to different or modified groups. Finally, we will synthesis or generate characteristics of studies contributing to each comparison by considering key PICOST characteristics across studies as wells important differences in the characteristics that could be possible effect modifiers. Synthesized data will be imported to STATA version 16.0 for analysis.

\section{DATA ANALYSIS.}




\section{Descriptive analysis.}

This will be done by use mean, median or proportion and a general narrative of all the synthesized data.

\section{Statistical analysis.}

For continuous variables, we will use mean differences in cases where the same units are used and standardized mean differences where the units used are different. Other alternative measures of effect will include risk ratios and risk differences. For categorical variables, we will use the odds ratio (OR) and their $95 \%$ confidence interval. We will use a random-effect model. This model assumes heterogeneity between the studies being combined that is studies are assumed different, and as such the differences in variation among studies will be thought to be due to not only random error but also between study variability in results. We will analyze the summarized data set to determine the $P$. falciparum genetic diversity and $\mathrm{MOI}$ as well as establish the genotyping approaches and methods of reporting it in Africa.

\section{Heterogeneity analysis.}

The Higgins $\left.\right|^{2}$ statistic will be used to predict whether the expected degree of heterogeneity is greater than the sampling error. Higgins values of less than $25 \%$ will be considered to show low heterogeneity, a value of $50 \%$ will be deemed average, and a value greater than $75 \%$ will indicate high heterogeneity. A low heterogeneity will be detected using a forest plot in case there is an overlap between the confidence intervals. Alternatively, Cochrane's $Q$ test (chi-squared), will be used to assess heterogeneity. If it's found to be less than 0.1 , then will be considered to show low heterogeneity.

\section{Meta-analysis.}

This will be conducted if heterogeneity is low. A Metaprop statistical program will be used to perform a meta-analysis. The pooled estimate of $P$. falciparum genetic diversity and $\mathrm{MOI}$, and genotyping approaches in Africa will be determined. This will be explained using a forest plot. The risk ratios or ORs and their $95 \%$ confidence intervals in each study as well as those calculated for all the included studies will be considered. A meta-regression analysis will also be performed to explain the low heterogeneity. This will enable the performance of a regression analysis of the pooled estimate for the covariance at the study level. Both univariate and multivariate regression analyses will be considered.

\section{Results presentation.}

A flowchart will be used to display the literature search and selection process according to the inclusion/exclusion criteria.

A table will be used to show a summary of the characteristics of the included studies.

The results of data analysis will be presented using a forest plot and funnel plot. 


\section{Discussion}

Plasmodium falciparum malaria remains a global challenge mostly in Sub-Saharan African countries. Individuals living in malaria-endemic areas are often infected with multiple genetically distinct clones of $P$. falciparum resulting in $\mathrm{MOI}$. The understanding of the distribution and dynamics of $P$. falciparum genetic diversity and $\mathrm{MOI}$ gives insights of the changing landscape of malaria transmission as well as evaluation of malaria control interventions.

This review aims to assess the $P$. falciparum genetic diversity, $\mathrm{MOI}$, genotyping approaches and methods of reporting it in Africa. In this systematic review, the search strategy will based on the keywords ' $P$. falciparum' and 'Genetic diversity' and 'Multiplicity of infection' and 'Genotyping approaches' and 'Africa' . Data extraction and analysis along with an in-depth quality assessment will be based on the GRADE approach so as to generate quality findings. The main analysis of this systematic review will incorporate only studies with a low or moderate risk of bias. We will exclude studies with a high risk of bias, as these studies may influence the precision of this review, presenting a potential problem. The anticipated limitation of the review is limited quality studies with direct measures of $P$. falciparum genetic diversity and MOI. To minimize publication bias, any determinant factor of the study outcome will have been investigated and reported by a large number of studies. The findings of this review will enhance the understanding of the distribution and dynamics of $P$. falciparum genetic diversity and $\mathrm{MOI}$. This is vital in identifying routes of transmission and gene flow patterns which are important in detecting the origin of $P$. falciparum infections, profiling malaria hot spot areas in Africa. The identification of malaria hotspot areas is important for the implementation of targeted interventions. Additionally, study findings will serve as baseline data for future studies on parasite population structure and antimalarial drug resistance surveillance across African countries.

\section{Study limitations.}

Limited quality studies with direct measures of $P$. falciparum genetic diversity and $\mathrm{MOI}$.

Use of weak and varied genotyping methods may hamper comparison of study outcomes.

\section{Declarations}

Ethical approval and consent to participate.

Not applicable.

\section{Availability of supporting data.}

Not applicable.

\section{Acknowledgments.}


The authors thank M/s Regina Ndagire and M/s Rachel Wangi Nante for their proofreading and constructive criticisms.

\section{Authors' information.}

Mwesigwa Alex is a PhD student at Makerere University College of health science. His PhD research project focuses on $P$. falciparum genetic diversity and multiplicity of infection in areas of varied malaria transmission intensity in Uganda. This will aid identification of malaria hot spot areas, understanding changes in malaria epidemiology to facilitate targeted control and elimination of malaria. Alex also works as an assistant lecturer in the department of microbiology and immunology at Kabale University.

Pauline Byakika-Kibibwika is an associate professor of medicine at makerere University College of health science. Prof Pauline is experienced in clinical care, research, occupational and travel medicine, capacity building, project development and management. She is the Director of Research and heads the Scientific Review Committee at the Department of Medicine. She is a member of the Accreditation Committee for Research Ethics Committees in Uganda, fellow of Uganda Academy of Sciences, and consultant for Ministry of Health and partners for Malaria and HIV/AIDS Control. At regional level, Pauline is a Commissioner on the East African Health Research Commission, where she contributes to decision making on health matters, research and evidence for knowledge generation, translation, policy formulations and practice.

Dr Joaniter I Nankabirwa and Dr. Sam L Nsobya are lecturers at Makerere University and researchers at Infectious diseases research collaboration of makerere University College of health sciences. They have vast experience in both malaria clinical and translational research.

Prof Charles and Dr Barbra Castelnuovo are well experience researchers at makerere University College of health sciences as well as reviewers for a number of journals.

\section{Authors' Contributions.}

AM, SLN, JIN and PBK conceived the idea, planned, and designed the study protocol.

AM designed and wrote the first draft.

CK, PBK and BC edited the protocol write-up.

PBK provided critical insights.

All the authors have approved and contributed to the final written manuscript.

Consent for publication. Not applicable.

Funding. No funding.

Competing interests. No competing interests. 


\section{References}

Abamecha, A., El-Abid, H., Yilma, D., Addisu, W., Ibenthal, A., Bayih, A. G., . . Abdissa, A. (2020). Genetic diversity and genotype multiplicity of Plasmodium falciparum infection in patients with uncomplicated malaria in Chewaka district, Ethiopia. Malaria Journal, 19, 1-9.

Carter, T. E., Malloy, H., Existe, A., Memnon, G., Victor, Y. S., Okech, B. A., \& Mulligan, C. J. (2015). Genetic diversity of Plasmodium falciparum in Haiti: insights from microsatellite markers. PLoS One, 10(10), e0140416.

Cochrane. (2014). Data collection forms for intervention reviews: Rcts and non-RCTs. Cocraine: London, UK.

Henning, L., Schellenberg, D., Smith, T., Henning, D., Alonso, P., Tanner, M., . . Felger, I. (2004). A prospective study of Plasmodium falciparum multiplicity of infection and morbidity in Tanzanian children. Transactions of the Royal Society of Tropical Medicine and Hygiene, 98(12), 687-694.

Higgins, J. P., Altman, D. G., Gøtzsche, P. C., Jüni, P., Moher, D., Oxman, A. D., . . Sterne, J. A. (2011). The Cochrane Collaboration's tool for assessing risk of bias in randomised trials. Bmj, 343.

Jensen, A. R., Adams, Y., \& Hviid, L. (2020). Cerebral Plasmodium falciparum malaria: The role of PfEMP1 in its pathogenesis and immunity, and PfEMP1-based vaccines to prevent it. Immunological reviews, 293(1), 230-252.

Jiang, H., Li, N., Gopalan, V., Zilversmit, M. M., Varma, S., Nagarajan, V., ... Henschen, B. (2011). High recombination rates and hotspots in a Plasmodium falciparum genetic cross. Genome biology, 12(4), 115.

Josling, G. A., \& Llinás, M. (2015). Sexual development in Plasmodium parasites: knowing when it's time to commit. Nature Reviews Microbiology, 13(9), 573-587.

Kidima, W., \& Nkwengulila, G. (2015). Plasmodium falciparum msp2 genotypes and multiplicity of infections among children under five years with uncomplicated malaria in Kibaha, Tanzania. Journal of parasitology research, 2015.

Kiwanuka, G. N. (2009). Genetic diversity in Plasmodium falciparum merozoite surface protein 1 and 2 coding genes and its implications in malaria epidemiology: a review of published studies from 19972007. Journal of vector borne diseases, 46(1), 1.

Landier, J., Parker, D. M., Thu, A. M., Lwin, K. M., Delmas, G., Nosten, F. H., . . Aung, E. P. (2018). Effect of generalised access to early diagnosis and treatment and targeted mass drug administration on Plasmodium falciparum malaria in Eastern Myanmar: an observational study of a regional elimination programme. The lancet, 391(10133), 1916-1926. 
Nabet, C., Doumbo, S., Jeddi, F., Konaté, S., Manciulli, T., Fofana, B., . . Ranque, S. (2016). Genetic diversity of Plasmodium falciparum in human malaria cases in Mali. Malaria Journal, 15(1), 1-10.

Niang, M., Thiam, L. G., Loucoubar, C., Sow, A., Sadio, B. D., Diallo, M., . . Toure-Balde, A. (2017). Spatiotemporal analysis of the genetic diversity and complexity of Plasmodium falciparum infections in Kedougou, southeastern Senegal. Parasites \& vectors, 10(1), 1-9.

Page, M. J., McKenzie, J. E., Bossuyt, P. M., Boutron, I., Hoffmann, T. C., Mulrow, C. D., . . Brennan, S. E. (2021). The PRISMA 2020 statement: an updated guideline for reporting systematic reviews. Bmj, 372.

Quagliato, L. A., Freire, R. C., \& Nardi, A. E. (2018). The role of acid-sensitive ion channels in panic disorder: a systematic review of animal studies and meta-analysis of human studies. Translational psychiatry, 8(1), 1-12.

Schünemann, H., Brożek, J., Guyatt, G., \& Oxman, A. (2013). Handbook for grading the quality of evidence and the strength of recommendations using the GRADE approach. Updated October, 2013.

Sondén, K., Doumbo, S., Hammar, U., Vafa Homann, M., Ongoiba, A., Traoré, B., . . Färnert, A. (2015). Asymptomatic multiclonal Plasmodium falciparum infections carried through the dry season predict protection against subsequent clinical malaria. The Journal of infectious diseases, 212(4), 608-616.

Sondo, P., Derra, K., Lefevre, T., Diallo-Nakanabo, S., Tarnagda, Z., Zampa, O., . . Ouedraogo, J.-B. (2019). Genetically diverse Plasmodium falciparum infections, within-host competition and symptomatic malaria in humans. Scientific reports, 9(1), 1-9.

Soulama, I., Nébié, I., Ouédraogo, A., Gansane, A., Diarra, A., Tiono, A. B., .. Taylor, W. R. (2009).

Plasmodium falciparum genotypes diversity in symptomatic malaria of children living in an urban and a rural setting in Burkina Faso. Malaria Journal, 8(1), 1-8.

Taylor, A. R., Schaffner, S. F., Cerqueira, G. C., Nkhoma, S. C., Anderson, T. J., Sriprawat, K., . . Buckee, C. O. (2017). Quantifying connectivity between local Plasmodium falciparum malaria parasite populations using identity by descent. PLoS genetics, 13(10), e1007065.

Wesolowski, A., Taylor, A. R., Chang, H.-H., Verity, R., Tessema, S., Bailey, J. A., . . Buckee, C. O. (2018). Mapping malaria by combining parasite genomic and epidemiologic data. BMC medicine, 16(1), 1-8.

WHO. (2018). World Malaria Report 2018 (World Health Organization, Geneva, Switzerland, 2018). In.

WHO. (2020). World malaria report 2020: 20 years of global progress and challenges.

\section{Figures}




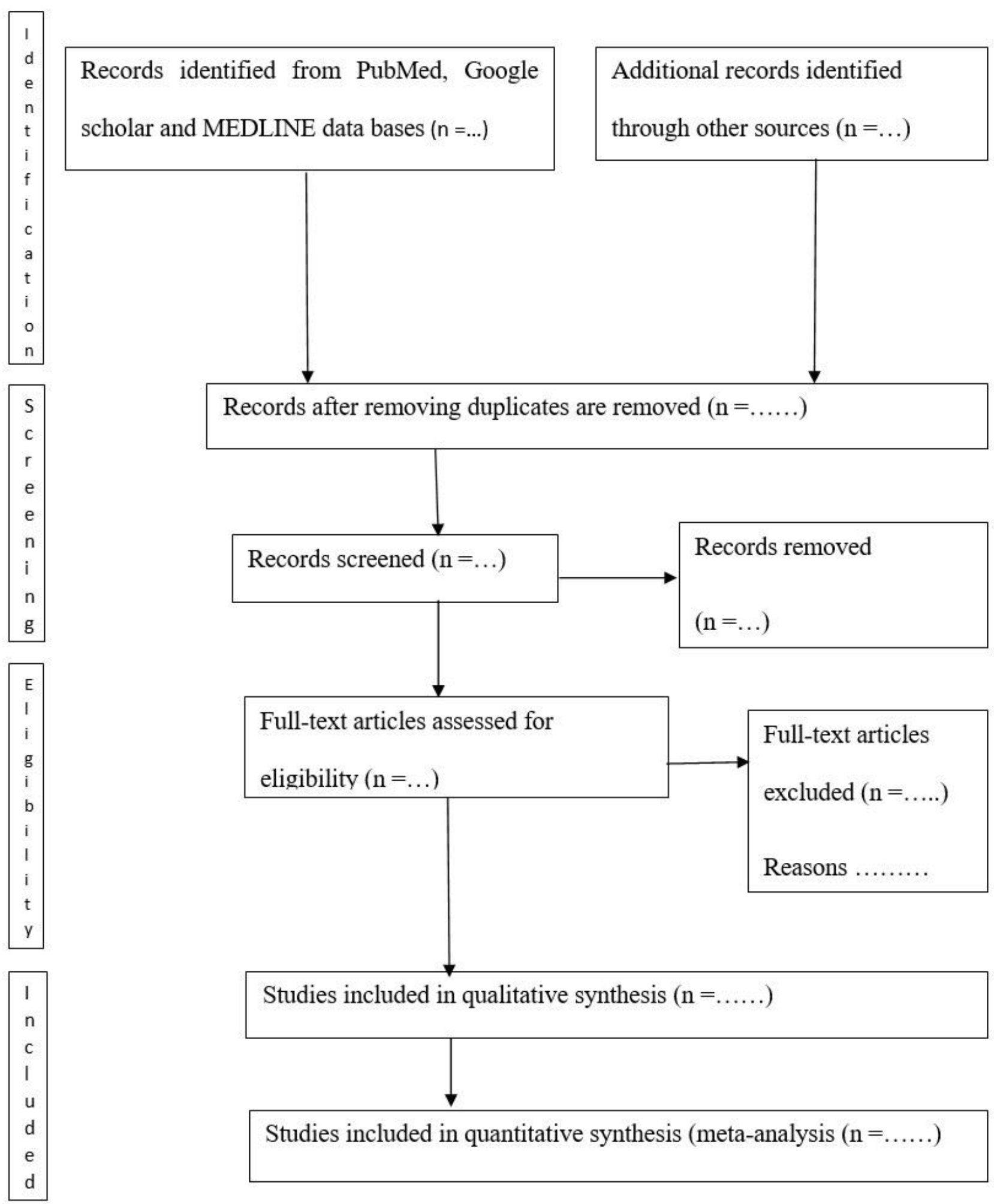

Figure 1

PRISMA flow diagram. Adapted from Quagliato, Freire, and Nardi (2018) 
An initial search in PubMed to identify the MeSH terms. We will use terms contained in the title like $P$. falciparum, genetic diversity, multiplicity of infection, genotyping approaches and Africa.

Use Boolean operator words "AND" to link unrelated MeSH terms, 'OR' for related terms to form a search string.

Apply the string in PubMed, Google scholar and MEDLIINE databases to obtain the articles.

Consider only articles from predefined study designs.

Add other articles from pre-determined sources.

The selection of studies will follow PRISMA guidelines.

\section{Figure 2}

Schematic process of search strategy according to PRISMA guidelines.

\section{Supplementary Files}

This is a list of supplementary files associated with this preprint. Click to download.

- PRISMAPchecklist.doc 